\title{
Model independent result on possible diurnal effect in DAMA/LIBRA-phase1
}

\author{
R. Bernabei ${ }^{1,2, a}$, P. Belli ${ }^{2}$, F. Cappella ${ }^{3,4}$, V. Caracciolo ${ }^{5}$, S. Castellano ${ }^{5}$, R. Cerulli ${ }^{5}$, C. J. Dai ${ }^{6}$, A. d'Angelo ${ }^{3,4}$,

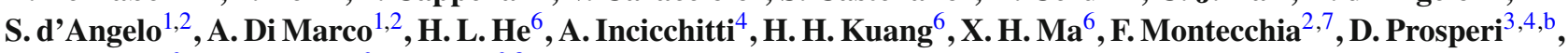 \\ X. D. Sheng ${ }^{6}$, R. G. Wang ${ }^{6}$, Z. P. Ye (6,8 $^{6}$ \\ ${ }^{1}$ Dip. di Fisica, Università di Roma "Tor Vergata”, 00133 Rome, Italy \\ 2 INFN, sez. Roma "Tor Vergata", 00133 Rome, Italy \\ ${ }^{3}$ Dip. di Fisica, Università di Roma "La Sapienza", 00185 Rome, Italy \\ ${ }^{4}$ INFN, sez. Roma, 00185 Rome, Italy \\ ${ }^{5}$ Laboratori Nazionali del Gran Sasso, INFN, Assergi, Italy \\ ${ }^{6}$ Institute of High Energy Physics, Chinese Academy of Sciences, P.O. Box 918/3, Beijing 100049, China \\ ${ }^{7}$ Dip. di Ingegneria Civile e Ingegneria Informatica, Università di Roma "Tor Vergata”, 00133 Rome, Italy \\ ${ }^{8}$ University of Jing Gangshan, Jiangxi, China
}

Received: 6 December 2013 / Accepted: 18 March 2014 / Published online: 27 March 2014

(C) The Author(s) 2014. This article is published with open access at Springerlink.com

\begin{abstract}
The results obtained in the search for possible diurnal effect in the single-hit low energy data collected by DAMA/LIBRA-phase 1 (total exposure 1.04 ton $\times$ year) deep underground at the Gran Sasso National Laboratory (LNGS) of the INFN are presented. At the present level of sensitivity the presence of any significant diurnal variation and of diurnal time structures in the data can be excluded for both the cases of solar and sidereal time. In particular, the diurnal modulation amplitude expected, because of the Earth diurnal motion, on the basis of the DAMA dark matter annual modulation results is below the present sensitivity.
\end{abstract}

\section{Introduction}

The present DAMA/LIBRA [1-8] experiment, as the former DAMA/NaI [8-11], has the main aim to investigate the presence of dark matter (DM) particles in the galactic halo by exploiting the model independent DM annual modulation signature (originally suggested in Ref. $[12,13]$ ). In particular, they have cumulatively reached a model independent evidence at $9.3 \sigma$ C.L. for the presence of DM particles in the galactic halo on the basis of the exploited DM annual modulation signature [4].

In the present work the DAMA/LIBRA-phase1 data (total exposure 1.04 ton $\times$ year) are analysed in terms of possi-

\footnotetext{
a e-mail: rita.bernabei@roma2.infn.it

${ }^{\mathrm{b}}$ Deceased
}

ble diurnal variation of the rate of the single-hit events ${ }^{1}$ in low energy regions, both where the DM annual modulation signal is observed (2-6 keV, see Ref. [2-4] and references therein) and in the region just above (6-14 keV) for comparison. Actually a diurnal effect with the sidereal time is expected for DM because of Earth rotation. In Sect. 2 the diurnal modulation of the DM signal as a function of the sidereal time due to Earth rotation velocity contribution will be discussed and some relevant formulae will be presented; this effect is model-independent and has several requirements as the DM annual modulation effect does. Thus, in Sect. 4 the data have been analyzed using the sidereal time referred to Greenwich, also sometimes called GMST. Since potential environmental backgrounds can be in principle correlated with the solar time, the analysis has been also performed in terms of solar time referred to the LNGS site.

\section{Expectation for DM diurnal effect because of the Earth rotation}

Let us now introduce an interesting model independent effect which can induce a diurnal variation of the counting rate of the single-hit events: the diurnal modulation of the DM signal as a function of the sidereal time due to Earth rotation velocity contribution [14]. As explained below, this effect is linked with the DM model independent annual modulation signature

\footnotetext{
1 i.e. those events where only one of the 25 detectors in DAMA/LIBRA fires; that is, each detector has all the others as anti-coincidence.
} 
(see also for example Refs. [15-18]). To explain this point in this section we describe all the components of the motion of a detector placed in a terrestrial laboratory with respect to an observer fixed in the galactic frame, including both the revolution of the Earth around the Sun and the rotation of the Earth around its axis.

As known, the velocity of the detector in the terrestrial laboratory can be expressed as following:

$\vec{v}_{l a b}(t)=\vec{v}_{L S R}+\vec{v}_{\odot}+\vec{v}_{r e v}(t)+\vec{v}_{r o t}(t)$,

where $\vec{v}_{L S R}$ is the velocity of the Local Standard of Rest (LSR) due to the rotation of the Galaxy; $\vec{v}_{\odot}$ is the Sun peculiar velocity with respect to LSR; $\vec{v}_{r e v}(t)$ is the velocity of the revolution of the Earth around the Sun and $\vec{v}_{\text {rot }}(t)$ is the velocity of the rotation of the Earth around its axis at the latitude and longitude of the laboratory. Using the galactic coordinate frame (that is $x$ axis towards the galactic center, $y$ axis following the rotation of the Galaxy and the $z$ axis towards the galactic North pole), we have $\vec{v}_{L S R}=\left(0, v_{0}, 0\right)$, with $v_{0}=(220 \pm 50) \mathrm{km} / \mathrm{s}[11,19-21]$ (uncertainty at $90 \%$ C.L.), and $\vec{v}_{\odot}=(9,12,7) \mathrm{km} / \mathrm{s}$ [22], while the revolution and rotation velocities of the Earth depend on the sidereal time, $t$.

Revolution of the Earth around the Sun. This motion can be easily worked out using the ecliptic coordinate system $\left(\hat{e}_{1}^{e c l}, \hat{e}_{2}^{e c l}, \hat{e}_{3}^{e c l}\right)$, where the $\hat{e}_{1}^{e c l}$ axis is directed towards the vernal equinox and $\hat{e}_{1}^{e c l}$ and $\hat{e}_{2}^{e c l}$ lie on the ecliptic plane. The right-handed convention is used. In the galactic coordinates, we can write ${ }^{2}$ :

$\hat{e}_{1}^{e c l}=(-0.05487,0.49411,-0.86767)$,

$\hat{e}_{2}^{e c l}=(-0.99382,-0.11100,-0.00035)$,

$\hat{e}_{3}^{e c l}=(-0.09648,0.86228,0.49715)$.

The ecliptic plane is tilted with respect to the galactic plane by $\approx 60^{\circ}$, as $\hat{e}_{3}^{e c l} \cdot(0,0,1)=0.49715$.

The motion of the Earth in the ecliptic plane can be described as:

$\vec{v}_{\text {rev }}(t)=V_{\text {Earth }}\left(\hat{e}_{1}^{e c l} \sin \lambda(t)-\hat{e}_{2}^{e c l} \cos \lambda(t)\right)$

where $V_{\text {Earth }}$ is the orbital velocity of the Earth, which has a weak dependence on time due to the ellipticity of the Earth orbital motion around the Sun; its value ranges between 29.3 $\mathrm{km} / \mathrm{s}$ and $30.3 \mathrm{~km} / \mathrm{s}$. For most purposes it can be assumed constant and equal to its mean value $\simeq 29.8 \mathrm{~km} / \mathrm{s}$. On the other hand, when more accurate calculations are necessary, the routines in Ref. [23] can be used: they also take into account the ellipticity of the Earth orbit and the gravitational influence

\footnotetext{
2 The coordinates of these versors are firstly worked out in the equatorial coordinate system by using the routines given in Ref. [23] and then in the galactic coordinate system by using the $R A$ and the $D E$ of the galactic North pole and of the galactic center (see also later).
}

of other celestial bodies (Moon, Jupiter, and etc.) ${ }^{3}$. Moreover, the phase in Eq. 3 can be written as $\lambda(t)=\omega\left(t-t_{\text {equinox }}\right)$; here $\omega=2 \pi / T$ with $T=1 \mathrm{y}, t$ is the sidereal time and $t_{\text {equinox }}$ is the spring equinox time $(\approx$ March 21$)$.

Rotation of the Earth around its axis. The simplest way to express this motion is in the equatorial coordinate system $\left(\hat{e}_{1}^{e c s}, \hat{e}_{2}^{e c s}, \hat{e}_{3}^{e c s}\right)$, where the $\hat{e}_{1}^{e c s}$ axis is directed towards the vernal equinox and $\hat{e}_{1}^{e c s}$ and $\hat{e}_{2}^{e c s}$ are on the equatorial plane; the $\hat{e}_{3}^{e c s}$ axis is towards the North pole. The right-handed convention is used. To work out the galactic coordinates of these versors, we use the equatorial coordinates of the galactic North pole: $R A=192^{\circ} .859508$ and $D E=27^{\circ} .128336$ ( $R A$ is the right ascension and $D E$ is declination); and of the galactic center: $R A=266^{\circ} .405100$ and $D E=-28^{\circ} .936175$, evaluated at the Epoch J2000.0. In the galactic coordinates, these versors can be written as:

$$
\begin{aligned}
& \hat{e}_{1}^{e c s}=(-0.05487,0.49411,-0.86767), \\
& \hat{e}_{2}^{e c s}=(-0.87344,-0.44483,-0.19808), \\
& \hat{e}_{3}^{e c s}=(-0.48384,0.74698,0.45599) .
\end{aligned}
$$

Therefore, we can write:

$\vec{v}_{r o t}(t)=-V_{r}\left(\hat{e}_{1}^{e c s} \sin \delta(t)-\hat{e}_{2}^{e c s} \cos \delta(t)\right)$

where $V_{r}$ is the rotational velocity of the Earth at the given latitude, $\phi_{0}$, of the laboratory: $V_{r}=V_{e q} \cos \phi_{0}$. The equatorial rotational velocity, $V_{e q}$, is equal to $0.4655 \mathrm{~km} / \mathrm{s}$. Hence, at LNGS $\left(\phi_{0}=42^{\circ} 27^{\prime} \mathrm{N}\right.$ and longitude $\left.\lambda_{0}=13^{\circ} 34^{\prime} \mathrm{E}\right)$ : $V_{r}=0.3435 \mathrm{~km} / \mathrm{s}$. The angle $\delta(t)=\omega_{\text {rot }}\left(t+\lambda_{0}\right)$, where $\omega_{r o t}=2 \pi / T_{d}$ with $T_{d}=1$ sidereal day.

\subsection{The time dependence of $\left|\vec{v}_{l a b}(t)\right|$}

In most evaluations of dark matter candidates the expected counting rate depends on the module of the detector's velocity in the Galaxy, $v_{l a b}(t)$. The time-independent contribution is $\left|\vec{v}_{S}\right|=\left|\vec{v}_{L S R}+\vec{v}_{\odot}\right| \approx 232 \pm 50 \mathrm{~km} / \mathrm{s}$, while a Taylor expansion can be performed in the smaller time-dependent contributions $\left|\vec{v}_{\text {rev }}(t)\right| \approx 30 \mathrm{~km} / \mathrm{s}$ and $\left|\vec{v}_{\text {rot }}(t)\right| \approx 0.34 \mathrm{~km} / \mathrm{s}$. Thus, to the first order, that reads:

$v_{l a b}(t) \simeq v_{s}+\hat{v}_{s} \cdot \vec{v}_{r e v}(t)+\hat{v}_{s} \cdot \vec{v}_{r o t}(t)$.

The higher order terms with no time dependence and with higher harmonics of $\vec{v}_{\text {rev }}(t): \frac{1}{2} \frac{V_{E a r t h}^{2}}{\left|\vec{v}_{s}\right|}-\frac{1}{2} \frac{\left(\hat{v}_{s} \cdot \vec{v}_{\text {rev }}(t)\right)^{2}}{\left|\vec{v}_{s}\right|}$, and the contributions arising from the ellipticity of the Earth orbit are omitted for simplicity, having frequencies well separated from the diurnal one.

\footnotetext{
${ }^{3}$ For completeness, we remind the discussion about the ellipticity of the Earth orbit in Ref. [18], which overcomes the description given in Ref. [24].
} 


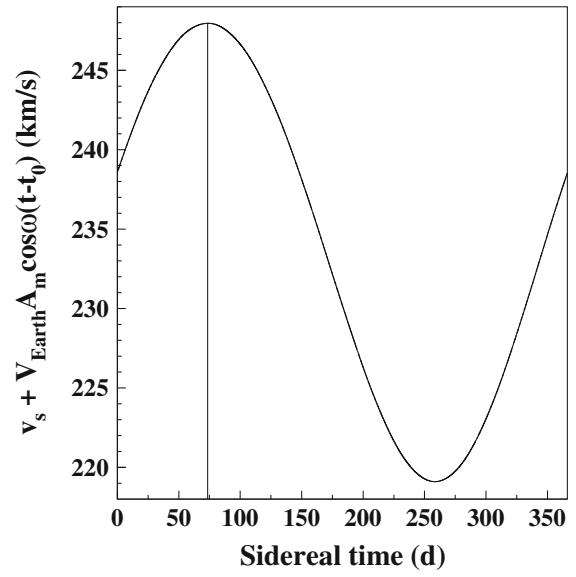

Fig. 1 Left velocity of the Earth in the galactic frame as a function of the sidereal time, with starting point March 21 (around spring equinox). The contribution of diurnal rotation (the third term in Eq. 6) has been dropped off. The maximum of the velocity (vertical line) is about 73 days after the spring equinox. Right sum of the Sun velocity in the galactic frame

The second term in Eq. 6 is responsible for the DM annual modulation of the signal and can be written as:

$\hat{v}_{s} \cdot \vec{v}_{\text {rev }}(t)=V_{\text {Earth }}\left(\hat{v}_{s} \cdot \hat{e}_{1}^{e c l} \sin \lambda(t)-\hat{v}_{s} \cdot \hat{e}_{2}^{e c l} \cos \lambda(t)\right)$.

Defining $\hat{v}_{s} \cdot \hat{e}_{1}^{e c l}=A_{m} \sin \beta_{m}$ and $-\hat{v}_{s} \cdot \hat{e}_{2}^{e c l}=A_{m} \cos \beta_{m}$ (equal to 0.465 and to 0.149 , respectively, for the case $v_{0}=$ $220 \mathrm{~km} / \mathrm{s}$ ), one can write:

$$
\begin{aligned}
\hat{v}_{s} \cdot \vec{v}_{r e v}(t) & =V_{\text {Earth }} A_{m} \cos \left(\lambda(t)-\beta_{m}\right) \\
& =V_{\text {Earth }} A_{m} \cos \left(\omega\left(t-t_{0}\right)\right),
\end{aligned}
$$

with $A_{m} \simeq 0.489$, this confirms that the ecliptic is tilted with respect to the galactic plane of $\simeq 60^{\circ}$, and $\beta_{m} \simeq 1.260 \mathrm{rad}$ (both values are calculated for $v_{0}=220 \mathrm{~km} / \mathrm{s}$ ). The phase of the DM annual modulation is determined at the time when the argument of cosine is null:

$t_{0}=t_{\text {equinox }}+\beta_{m} / \omega=t_{\text {equinox }}+73.25$ solar days,

for $v_{0}=220 \mathrm{~km} / \mathrm{s}$; it corresponds to $\approx$ June 2 nd (see Fig. 1 , left). The term in the previous equation ranges from 71.76 solar days (for $v_{0}=170 \mathrm{~km} / \mathrm{s}$ ) to 74.20 solar days (for $v_{0}=$ $270 \mathrm{~km} / \mathrm{s}$ ).

The same procedure can be followed to determine the phase of the diurnal modulation due to the Earth rotation around its axis, described by the third term in Eq. 6:

$\hat{v}_{s} \cdot \vec{v}_{r o t}(t)=-V_{r}\left(\hat{v}_{s} \cdot \hat{e}_{1}^{e c s} \sin \delta(t)-\hat{v}_{s} \cdot \hat{e}_{2}^{e c s} \cos \delta(t)\right)$.

Defining $\hat{v}_{s} \cdot \hat{e}_{1}^{e c s}=-A_{d} \sin \beta_{d}$ and $\hat{v}_{s} \cdot \hat{e}_{2}^{e c s}=A_{d} \cos \beta_{d}$ (equal to 0.465 and to -0.484 , respectively, for the case $v_{0}=$ $220 \mathrm{~km} / \mathrm{s}$ ), one can write:

$\hat{v}_{s} \cdot \vec{v}_{r o t}(t)=V_{r} A_{d} \cos \left(\delta(t)-\beta_{d}\right)=V_{r} A_{d} \cos \left[\omega_{r o t}\left(t-t_{d}\right)\right]$,

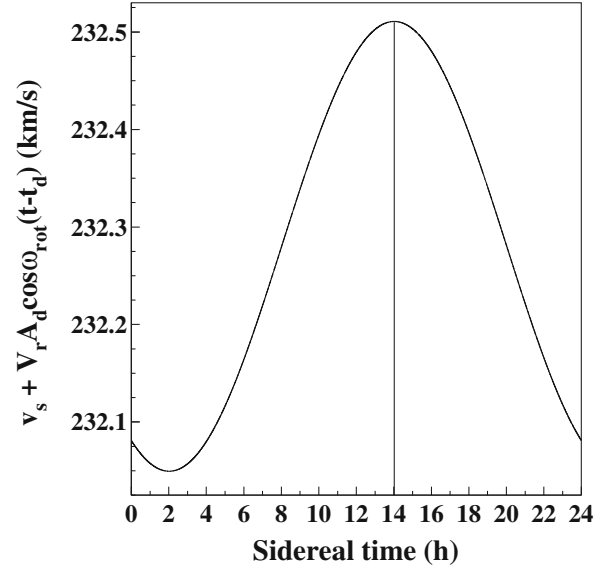

$\left(v_{s}\right)$ and of the rotation velocity of a detector at LNGS $\left(\hat{v}_{s} \cdot \vec{v}_{\text {rot }}(t)\right)$ as a function of the sidereal time. The maximum of the velocity is about at $14 \mathrm{~h}$ (vertical line). These velocities have been calculated assuming $v_{0}=220 \mathrm{~km} / \mathrm{s}$ by using the routines of Ref. [23]

with $A_{d} \simeq 0.671$ and $\beta_{d} \simeq 3.907$ rad (both values are calculated for $v_{0}=220 \mathrm{~km} / \mathrm{s}$ ). The phase of the DM diurnal modulation is determined at the time when the argument of cosine is null:

$t_{d}=\beta_{d} / \omega_{\text {rot }}-\lambda_{0}$.

It corresponds to $t_{d} \simeq 14.02 \mathrm{~h}$ sidereal time for the case of a detector at the Gran Sasso longitude ${ }^{4}$ and $v_{0}=220 \mathrm{~km} / \mathrm{s}$ (see Fig. 1, right); actually this value ranges from $13.94 \mathrm{~h}$ $\left(v_{0}=170 \mathrm{~km} / \mathrm{s}\right)$ to $14.07 \mathrm{~h}\left(v_{0}=270 \mathrm{~km} / \mathrm{s}\right)$.

Finally, the detector's velocity in the Galaxy can be written as:

$$
\begin{aligned}
v_{\text {lab }}(t) \simeq & v_{s}+V_{\text {Earth }} A_{m} \cos \left[\omega\left(t-t_{0}\right)\right] \\
& +V_{r} A_{d} \cos \left[\omega_{\text {rot }}\left(t-t_{d}\right)\right] .
\end{aligned}
$$

\subsection{The time dependence of the counting rate}

In most evaluations of dark matter candidates the expected counting rate depends on the module of the detector's velocity in the Galaxy, $v_{l a b}(t)$. Applying a Taylor expansion, as done in the previous section, the expected signal counting rate in a given $k$-th energy bin can be written as:

$$
\begin{aligned}
S_{k}\left[v_{l a b}(t)\right] \simeq & S_{k}\left[v_{s}\right]+\left[\frac{\partial S_{k}}{\partial v_{l a b}}\right]_{v_{s}}\left[V_{\text {Earth }} A_{m} \cos \omega\left(t-t_{0}\right)\right. \\
& \left.+V_{r} A_{d} \cos \omega_{\text {rot }}\left(t-t_{d}\right)\right] .
\end{aligned}
$$

The higher order terms with no time dependence and with higher harmonics of $\omega$ are omitted for simplicity. In Eq. 8 the

\footnotetext{
${ }^{4}$ Note that in terms of local sidereal time the phase of the DM diurnal modulation is given by $t_{d}+\lambda_{0}=\beta_{d} / \omega_{\text {rot }} \simeq 14.92 \mathrm{~h}$ and it is the same for each laboratory independently of its longitude.
} 
first term is the constant part of the signal $\left(S_{0}\right)$, the second term is the annual modulation term with amplitude $S_{m}=$ $\left[\frac{\partial S_{k}}{\partial v_{\text {lab }}}\right]_{v_{s}} V_{E a r t h} A_{m}$, and the third term provides a diurnal modulation with amplitude $S_{d}=\left[\frac{\partial S_{k}}{\partial v_{l a b}}\right]_{v_{s}} V_{r} A_{d}$.

The interest in this signature is that the ratio $R_{d y}$ of this diurnal modulation amplitude over the annual modulation amplitude is a model independent constant; considering the LNGS latitude one has:

$R_{d y}=\frac{S_{d}}{S_{m}}=\frac{V_{r} A_{d}}{V_{\text {Earth }} A_{m}} \simeq 0.016$

Taking into account $R_{d y}$ and the annual modulation effect evidenced by DAMA/LIBRA-phase 1 for single-hit events in the low energy region, it is possible to derive the diurnal modulation amplitude expected for the same data. In particular, when considering the (2-6) keV energy interval, the observed annual modulation amplitude in DAMA/LIBRAphase 1 is: $(0.0097 \pm 0.0013) \mathrm{cpd} / \mathrm{kg} / \mathrm{keV}$ [4] and the expected value of the diurnal modulation amplitude is $\simeq$ $1.5 \times 10^{-4} \mathrm{cpd} / \mathrm{kg} / \mathrm{keV}$.

\section{The experimental set-up}

The results presented in the following have been obtained by analysing the data collected in seven annual cycles by DAMA/LIBRA-phase1 (1.04 ton $\times$ year exposure) [2-4] at LNGS. The description, radiopurity and main features of the DAMA/LIBRA-phase1 setup are discussed in details in the dedicated Ref. [1]. The sensitive part is made of 25 highly radiopure $\mathrm{NaI}(\mathrm{Tl})$ crystal scintillators organized in a $(5 \times 5)$ matrix; each $\mathrm{NaI}(\mathrm{Tl})$ detector has $9.70 \mathrm{~kg}$ mass and a size of $(10.2 \times 10.2 \times 25.4) \mathrm{cm}^{3}$. The bare crystals are enveloped in Tetratec-teflon foils and encapsulated in radiopure OFHC $\mathrm{Cu}$ housing. In each detector two $10 \mathrm{~cm}$ long special quartz light guides act also as optical windows on the two end faces of the crystal and are coupled to two low background photomultipliers (PMT) working in coincidence at single photoelectron level. The detectors are housed in a sealed low-radioactive copper box installed in the center of a low-radioactive $\mathrm{Cu} / \mathrm{Pb} / \mathrm{Cd}$-foils/polyethylene/paraffin shield; moreover, about $1 \mathrm{~m}$ concrete (made from the Gran Sasso rock material) almost fully surrounds (mostly outside the barrack) this passive shield, acting as a further neutron moderator. The copper box is maintained in HP Nitrogen atmosphere in slightly overpressure with respect to the external environment; it is part of the threefold-level sealing system which excludes the detectors from the environmental air of the underground laboratory. The light response of the detectors in DAMA/LIBRA-phase1 typically ranges from 5.5 to 7.5 photoelectrons $/ \mathrm{keV}$, depending on the detector. The hardware threshold of each PMT is at single photo- electron, while a software energy threshold of $2 \mathrm{keV}$ electron equivalent (hereafter $\mathrm{keV}$ ) is used [1]. Energy calibration with $\mathrm{X}$-rays $/ \gamma$ sources are regularly carried out in the same running condition down to few $\mathrm{keV}$ [1]. Moreover, the whole DAMA/LIBRA installation is under air conditioning to assure a suitable and stable working temperature for the electronics; in addition, the huge heat capacity of the multiton passive shield $\left(\simeq 10^{6} \mathrm{cal} /{ }^{\circ} \mathrm{C}\right)$ further assures a relevant stability of the detectors' operating temperature. The DAQ system records both single-hit events (where just one of the detectors fires) and multiple-hit events (where more than one detector fire) up to the $\mathrm{MeV}$ region despite the optimization is performed for the lowest one. A hardware/software system is operative to monitor the running conditions, and selfcontrolled computer processes automatically control several parameters and manage alarms. For the radiopurity, the electronic chain, the data acquisition system and for all the other details see Ref. [1]; for completeness we recall that during DAMA/LIBRA-phase1 new transient digitizers and DAQ system have been installed at fall 2008 before the start of the sixth annual cycle.

\section{Model independent experimental results}

In order to point out the presence of a possible diurnal effect, the low energy single-hit DAMA/LIBRA-phase1 data have been grouped in 1 hour bin using either the sidereal or solar time, respectively. We define $N_{i, d}^{(j k y)}$ as the number of events collected in the $i$ th hour of the day $d$ (for the two cases of sidereal and solar time); as regards the other indexes: i) $j$ identifies the detector; ii) $k$ identifies the energy bin within the considered energy interval; iii) $y$ identifies the annual cycle. Hence, the single-hit rate in $i$ th hour is written as:

$r_{i}^{(j k y)}=\frac{\sum_{d} N_{i, d}^{(j k y)}}{\sum_{d} M_{j} \Delta t_{i, d}^{(y)} \Delta E \epsilon^{(j k y)}}$,

where $M_{j}$ is the mass of the $j$ th detector, $\Delta t_{i, d}^{(y)}$ is the detector running time during the $i$ th hour of the $d$ th day of the $y$ th annual cycle, $\Delta E$ is the energy bin and $\epsilon^{(j k y)}$ is the overall efficiency [1].

Therefore, the residual rate has been calculated according to: $\left\langle r_{i}^{(j k y)}-f l a t^{(j k y)}\right\rangle_{j k y}$, where the average is made on all the detectors ( $j$ index), on all the considered energy bins ( $k$ index), and on all the DAMA/LIBRA-phase 1 annual cycles ( $y$ index). The $f_{l a t}{ }^{(j k y)}$ is the rate averaged over the day: flat $^{(j k y)}=\left\langle r_{i}^{(j k y)}\right\rangle_{i}$, which is of the order of $\approx 1 \mathrm{cpd} / \mathrm{kg} / \mathrm{keV}$ [1]. Here, the possible time-dependent contribution due to the annual modulation signal is not considered since it is washed out by the almost uniform data collection along the day and along the year; its estimated effect is much lower than $\simeq 10^{-4}$ of flat $^{(j k y)}$. 
Fig. 2 Experimental model-independent diurnal residual rate of the single-hit scintillation events, measured by DAMA/LIBRA-phase 1 in the (2-4), (2-5) and (2-6) keV energy intervals as a function of the hour of the solar (left) and sidereal (right) day. The experimental points present the errors as vertical bars and the associated time bin width $(1 \mathrm{~h})$ as horizontal bars. The cumulative exposure is 1.04 ton $x$ year. See text
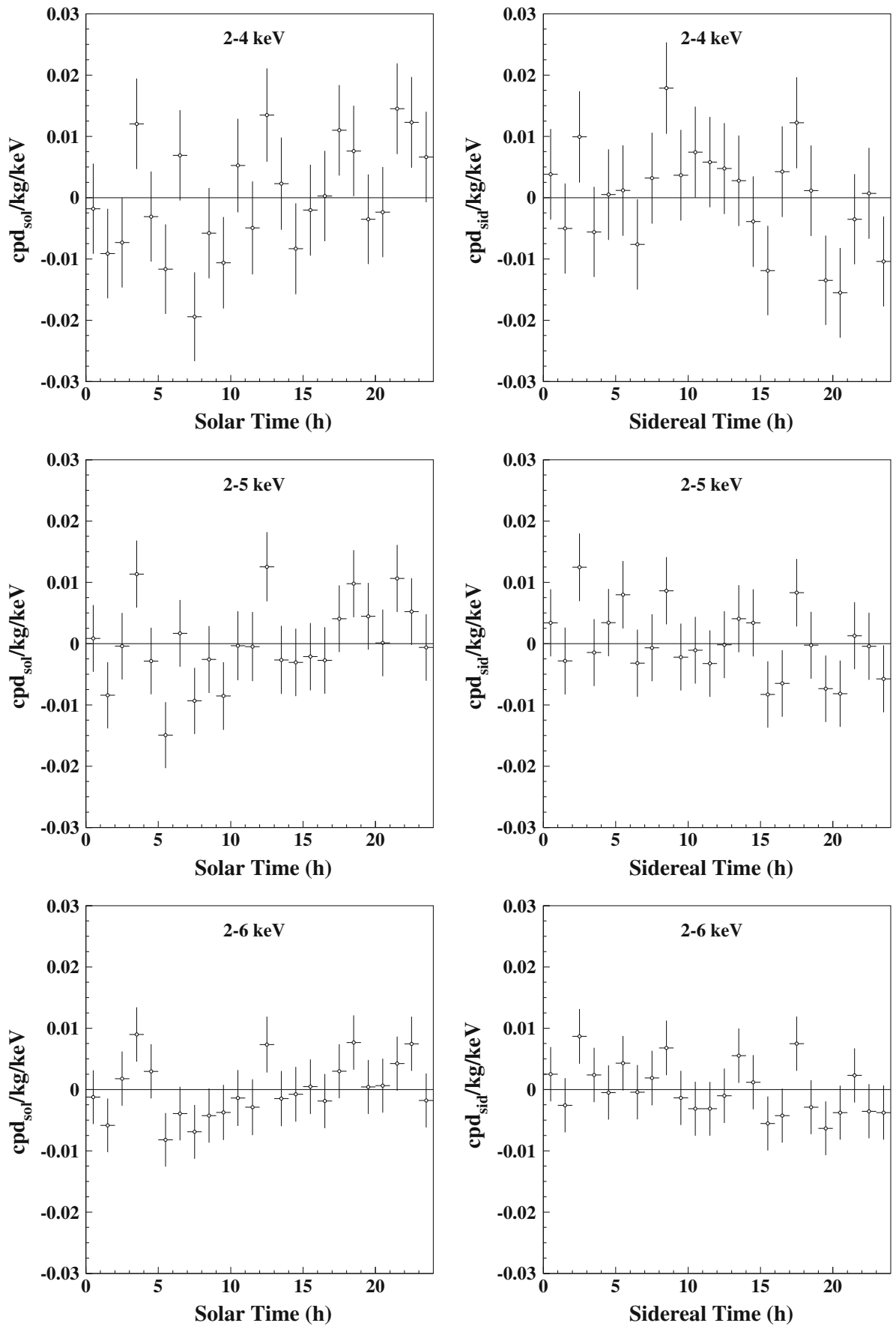

Figure 2 shows the time and energy behavior of the experimental residual rates of single-hit events both as a function of solar (left) and of sidereal (right) time, in the (2-4), (2-5) and (2-6) keV energy intervals ${ }^{5}$ and in Fig. 3 those in the (6-14) $\mathrm{keV}$ interval for the solar (left) and sidereal (right) case. The used time bin is 1 (either solar or sidereal, respectively) hour.

\footnotetext{
5 We recall that the annual modulation signal has been pointed out only in these energy intervals; see Ref. [2-4] and references therein.
}

The null hypothesis (absence of residual rate diurnal variation) has been tested by a $\chi^{2}$ test, obtaining the results given in Table 1; there the upper tail probabilities (P-values), calculated by the standard $\chi^{2}$ distribution, are also reported. Thus, no diurnal variation with a significance of $95 \%$ C.L. is found.

In addition to the $\chi^{2}$ test, another independent statistical test has been applied: the run test (see e.g. Ref. [25]); it verifies the hypothesis that the positive and negative data points are randomly distributed. The lower tail probabilities 
Fig. 3 Experimental model-independent diurnal residual rate of the single-hit scintillation events, measured by DAMA/LIBRA-phase1 in the (6-14) keV energy interval as a function of the hour of the solar (left) and sidereal (right) day. The experimental points present the errors as vertical bars and the associated time bin width $(1 \mathrm{~h})$ as horizontal bars. The cumulative exposure is 1.04 ton $x$ year. See text
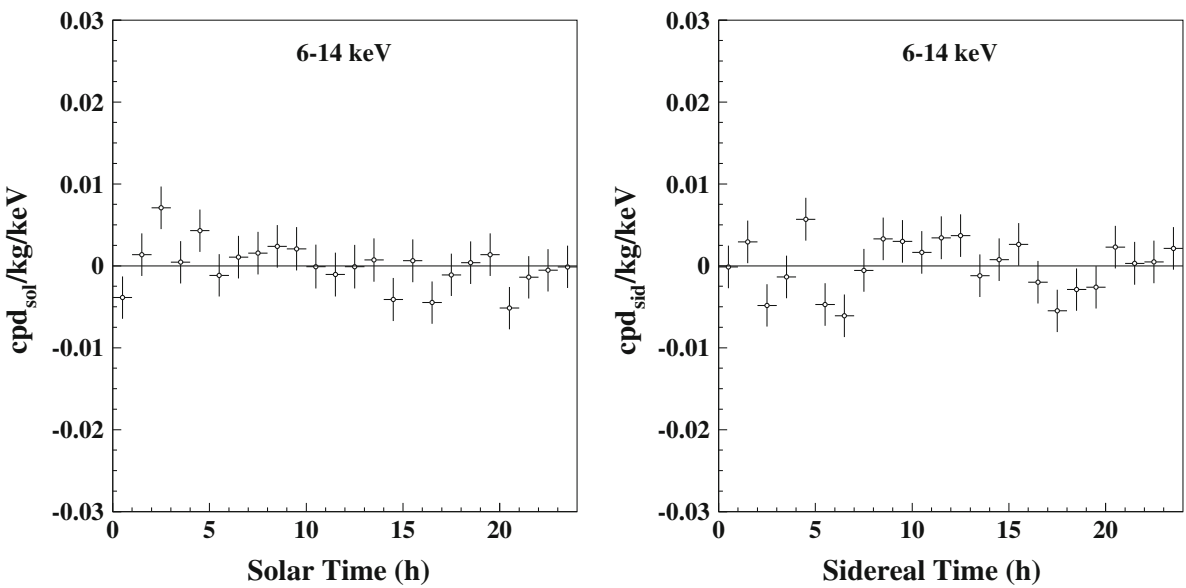

Table 1 Test of absence of diurnal effect in the DAMA/LIBRA-phase 1 data. The $\mathrm{P}$ values, calculated by the standard $\chi^{2}$ distribution, are also shown. As it can be seen, the $\chi^{2}$ test applied to the data supports the hypothesis that the residual rates are simply fluctuating around zero

\begin{tabular}{lll}
\hline $\begin{array}{l}\text { Energy } \\
(\mathrm{keV})\end{array}$ & Solar time & Sidereal time \\
\hline $2-4$ & $\chi^{2} /$ d.o.f. $=35.2 / 24 \rightarrow \mathrm{P}=7 \%$ & $\chi^{2} /$ d.o.f. $=28.7 / 24 \rightarrow \mathrm{P}=23 \%$ \\
$2-5$ & $\chi^{2} /$ d.o.f. $=35.5 / 24 \rightarrow \mathrm{P}=6 \%$ & $\chi^{2} /$ d.o.f. $=24.0 / 24 \rightarrow \mathrm{P}=46 \%$ \\
$2-6$ & $\chi^{2} /$ d.o.f. $=25.8 / 24 \rightarrow \mathrm{P}=36 \%$ & $\chi^{2} /$ d.o.f. $=21.2 / 24 \rightarrow \mathrm{P}=63 \%$ \\
$6-14$ & $\chi^{2} /$ d.o.f. $=25.5 / 24 \rightarrow \mathrm{P}=38 \%$ & $\chi^{2} /$ d.o.f. $=35.9 / 24 \rightarrow \mathrm{P}=6 \%$ \\
\hline
\end{tabular}

are equal to: $43,18,7$ and $26 \%$ in the (2-4), (2-5), (2-6) and (6-14) $\mathrm{keV}$ energy region, respectively, for the solar case and $54,84,78$ and $16 \%$ in the (2-4), (2-5), (2-6) and (6-14) keV energy region, respectively, for the sidereal case. Thus, in conclusion the presence of any significant diurnal variation and of time structures can be excluded at the reached level of sensitivity (see e.g. the error bars in Fig. 2).

\subsection{Comparison with expectation for DM diurnal effect}

When considering the DM diurnal effect due to the Earth rotation around its axis described in Sect. 2, only an upper limit can be derived. In particular, the residual rates of the single-hit events in the (2-4), (2-5), (2-6) and (6-14) keV energy intervals as a function of the sidereal time (see Figs. 2, right and 3, right) have been fitted with a cosine function with amplitude $A_{d}^{\text {exp }}$ as free parameter, period fixed at $24 \mathrm{~h}$ and phase at $14 \mathrm{~h}$. The results are reported in Table 2: all the diurnal modulation amplitudes are compatible with zero.

Figure 4 shows the diurnal modulation amplitudes, $A_{d}$, as function of energy (the energy bin is $1 \mathrm{keV}$ ) obtained by fitting the single-hit residual rate of the entire DAMA/LIBRAphase 1 as function of the sidereal time, with the formula $A_{d} \cos \left[\omega_{r o t}\left(t-t_{d}\right)\right]$. The period is fixed at $24 \mathrm{~h}$ and the phase at $14 \mathrm{~h}$, as expected for the DM diurnal effect (see
Table 2 Diurnal modulation amplitudes, $A_{d}^{\exp }$, for each considered energy interval obtained by fitting the single-hit residual rate of the entire DAMA/LIBRA-phase 1 as function of the sidereal time, (see Figs. 2 , right, 3 , right) with the formula $A_{d}^{\text {exp }} \cos \left[\omega_{\text {rot }}\left(t-t_{d}\right)\right]$. The amplitude $A_{d}^{e x p}$ is a free parameter, while the period is fixed at $24 \mathrm{~h}$ and the phase at $14 \mathrm{~h}$, as expected for the DM diurnal effect. The corresponding $\chi^{2}$ values of each fit and the P-values are also reported

\begin{tabular}{lrrc}
\hline Energy $(\mathrm{keV})$ & $A_{d}^{\text {exp }}(\mathrm{cpd} / \mathrm{kg} / \mathrm{keV})$ & $\chi^{2} /$ d.o.f. & $\mathrm{P}(\%)$ \\
\hline $2-4$ & $(2.0 \pm 2.1) \times 10^{-3}$ & $27.8 / 23$ & 22 \\
$2-5$ & $-(1.4 \pm 1.6) \times 10^{-3}$ & $23.2 / 23$ & 45 \\
$2-6$ & $-(1.0 \pm 1.3) \times 10^{-3}$ & $20.6 / 23$ & 61 \\
$6-14$ & $(5.0 \pm 7.5) \times 10^{-4}$ & $35.4 / 23$ & 5 \\
\hline
\end{tabular}

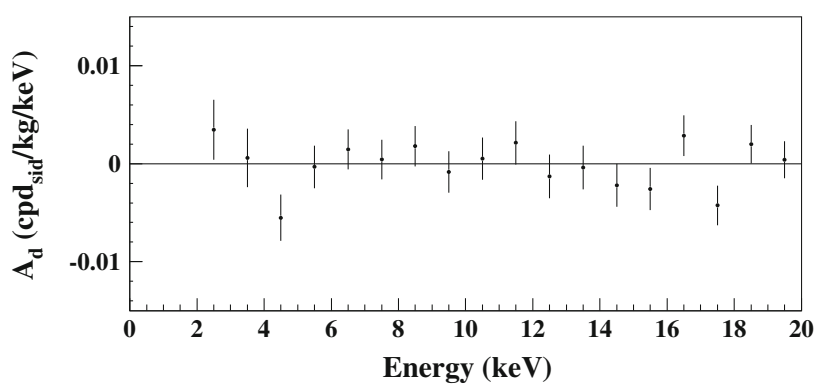

Fig. 4 Diurnal modulation amplitudes, $A_{d}$, as function of energy (the energy bin is $1 \mathrm{keV}$ ) obtained by fitting the single-hit residual rate of the entire DAMA/LIBRA-phase 1 as function of the sidereal time, with the formula $A_{d} \cos \left[\omega_{\text {rot }}\left(t-t_{d}\right)\right]$. The amplitude $A_{d}$ is a free parameter, while the period is fixed at $24 \mathrm{~h}$ and the phase at $14 \mathrm{~h}$, as expected for the DM diurnal effect. The $A_{d}$ values are compatible with zero, having random fluctuations around zero with $\chi^{2}$ equal to 19.5 for 18 degrees of freedom. The cumulative exposure is 1.04 ton $\times$ year. See text

above). The $A_{d}$ values are compatible with zero, having random fluctuations around zero with $\chi^{2}$ equal to 19.5 for 18 degrees of freedom.

In order to compare the experimental data with the DM diurnal effect due to the Earth rotation around its axis described in Sect. 2, the (2-6) keV energy interval is taken 
into account for simplicity. From Table 2 one can obtain $A_{d}^{\text {exp }}=-(1.0 \pm 1.3) \times 10^{-3} \mathrm{cpd} / \mathrm{kg} / \mathrm{keV}\left(\chi^{2} /\right.$ d.o.f. $=$ 20.6/23). Following the Feldman-Cousins [26] procedure an upper limit can be obtained for the measured diurnal modulation amplitude: $A_{d}^{\text {exp }}<1.2 \times 10^{-3} \mathrm{cpd} / \mathrm{kg} / \mathrm{keV}(90 \%$ C.L.); thus, the present experimental sensitivity is larger than the expected diurnal modulation amplitude $(\simeq 1.5 \times$ $10^{-4} \mathrm{cpd} / \mathrm{kg} / \mathrm{keV}$ ) derived above from the DAMA/LIBRAphase 1 observed effect.

In conclusion, it will be possible to investigate this diurnal effect with adequate sensitivity only when a much larger exposure will be available, provided a suitable control of the running parameters at the needed level. On the other hand, better sensitivities can also be achieved by lowering the software energy threshold; in fact an almost exponential rising of the signal rate is expected at lower energy for some DM candidates. This is one of the goals of the presently running DAMA/LIBRA-phase2.

\subsection{Comparison with any hypothetical diurnal effects} with cosine behaviour

In order to leave to the reader the possibility to compare the data with possible exotic models, the experimental residual rates of the single-hit events as function of both solar and sidereal time have been compared with a cosine function with a free phase. For this purpose the residual rate for each energy bin of $1 \mathrm{keV}$ has been fitted with the formula $A_{d} \cos \left[\omega_{\text {rot }}(t-\right.$ $\left.t_{d}^{*}\right)$ ]. The free parameters of the fit are the 18 modulation amplitudes (one for each energy bin) and the phase $t_{d}^{*}$. The period is fixed at $24 \mathrm{~h}$. The results are reported in Fig. 5 for both solar and sidereal time cases.

The $A_{d}$ values are compatible with zero, having random fluctuations around zero with $\chi^{2}$ equal to 24.2 and 25.4 (18 degrees of freedom) for the solar time and sidereal time, respectively. The best fit values for the phase are $t_{d}^{*}=(6.1 \pm$ $1.1) \mathrm{h}$ and $t_{d}^{*}=(10.7 \pm 1.1) \mathrm{h}$ for the solar time and sidereal time, respectively.

\subsection{Comparison with possible diurnal effects induced by cosmic rays}

Solar and sidereal diurnal modulation of the underground muon rate at LNGS have been searched for by the MACRO experiment computing hourly deviations of the muon rate from 6 month averages [27]. Statistically significant diurnal modulations with the solar and the sidereal periods have been pointed out: their amplitudes are $<0.1 \%$, at the limit of the detector statistics. The solar diurnal modulation is due to the diurnal atmospheric temperature variations at $20 \mathrm{~km}$, i.e. the altitude of primary cosmic ray interactions with the atmosphere; the sidereal diurnal modulation is due to the
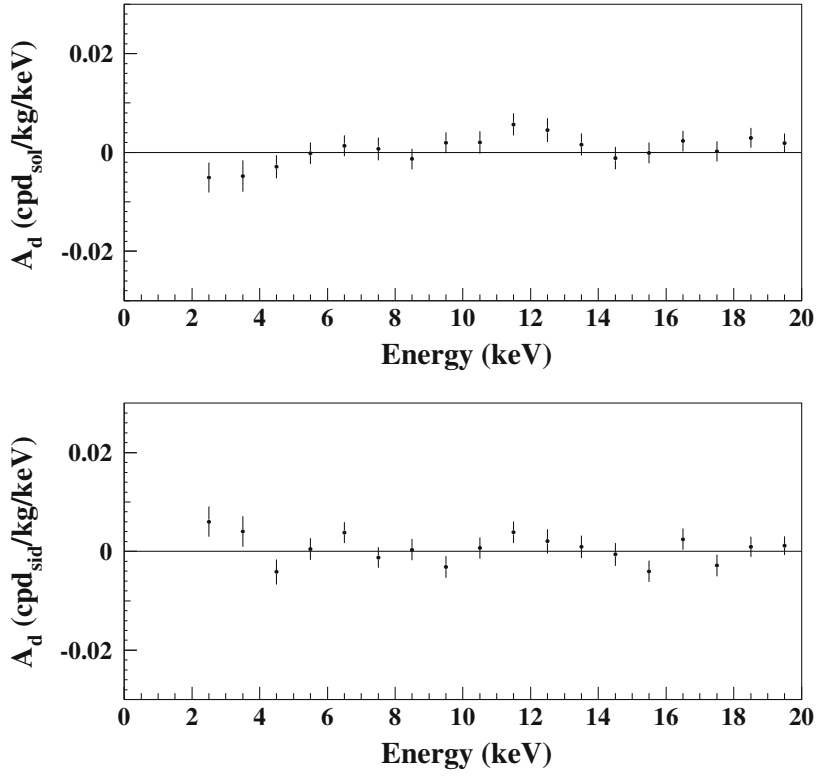

Fig. 5 Diurnal modulation amplitudes, $A_{d}$, as function of energy (the energy bin is $1 \mathrm{keV}$ ) obtained by fitting the single-hit residual rate of the entire DAMA/LIBRA-phase 1 with the formula $A_{d} \cos \left[\omega_{\text {rot }}\left(t-t_{d}^{*}\right)\right]$. The free parameters of the fit are the 18 modulation amplitudes (one for each energy bin) and the phase $t_{d}^{*}$. The results are reported for the solar time (top) and sidereal time (bottom) cases. The $A_{d}$ values are compatible with zero, having random fluctuations around zero with $\chi^{2}$ equal to 24.2 and 25.4 (18 degrees of freedom) for the solar time and sidereal time, respectively. The best fit values for the phase are $t_{d}^{*}=(6.1 \pm 1.1) \mathrm{h}$ and $t_{d}^{*}=(10.7 \pm 1.1) \mathrm{h}$ for the solar time and sidereal time, respectively. The cumulative exposure is 1.04 ton $\times$ year. See text

Compton-Getting modulation due to solar system motion relative to the local standard of rest.

Thus, we have estimated consistency of the result, obtained with the presently reached sensitivity, with these known effects. The measured single-hit event counting rate of DAMA/LIBRA-phase1 in the low energy region is of the order of $\approx 1 \mathrm{cpd} / \mathrm{kg} / \mathrm{keV}$ [1] and, as shown in Ref. [6], the contribution due to muons surviving the Gran Sasso mountain or related particles is very small $(\ll 1 \mathrm{cpd} / \mathrm{kg} / \mathrm{keV})$. Therefore, the effect due to diurnal variation of muon flux is expected to be $\ll 10^{-3} \mathrm{cpd} / \mathrm{kg} / \mathrm{keV}$, that is well below the present experimental sensitivity (see e.g. the error bars in Fig. 2).

\section{Conclusions}

The low energy (2-6) keV single-hit data collected in the whole DAMA/LIBRA-phase1 (7 annual cycles; exposure: 1.04 ton $\times$ year) [2-4] have been analyzed in terms of diurnal effects. At the present level of sensitivity the presence of any significant diurnal variation and of diurnal time structures in the data can be excluded for both the cases of solar and sidereal time. In particular, the diurnal modulation ampli- 
tude expected-because of the Earth diurnal motion—on the basis of the DAMA DM annual modulation results is below the present sensitivity; it will be possible to investigate this diurnal effect with adequate sensitivity only when a much larger exposure will be available, provided a suitable control of the running parameters at the needed level. At present DAMA/LIBRA is continuously running in its new configuration (named DAMA/LIBRA-phase2) with a lower software energy threshold [5] which also can offer an alternative possibility to increase sensitivity to such an effect.

Acknowledgments It is a pleasure to thank Mr. A. Bussolotti and A. Mattei for their qualified technical work.

Open Access This article is distributed under the terms of the Creative Commons Attribution License which permits any use, distribution, and reproduction in any medium, provided the original author(s) and the source are credited.

Funded by $\mathrm{SCOAP}^{3}$ / License Version CC BY 4.0.

\section{References}

1. R. Bernabei et al., Nucl. Instr. Meth. A 592, 297 (2008)

2. R. Bernabei et al., Eur. Phys. J. C 56, 333 (2008)

3. R. Bernabei et al., Eur. Phys. J. C 67, 39 (2010)
4. R. Bernabei et al., Eur. Phys. J. C 73, 2648 (2013). [arXiv:1308. 5109]

5. R. Bernabei et al., J. Instr. 7, P03009 (2012)

6. R. Bernabei et al., Eur. Phys. J. C 72, 2064 (2012)

7. R. Bernabei et al., Int. J. Mod. Phys. A 28, 1330022 (2013)

8. http://people.roma2.infn.it/dama/

9. R. Bernabei el al., La Rivista del Nuovo Cimento 26(1), 1-73 (2003)

10. R. Bernabei et al., Int. J. Mod. Phys. D 13, 2127 (2004)

11. P. Belli et al., Phys. Rev. D 61, 023512 (2000)

12. K.A. Drukier et al., Phys. Rev. D 33, 3495 (1986)

13. K. Freese et al., Phys. Rev. D 37, 3388 (1988)

14. R. Bernabei et al., Phys. Atom. Nucl. 72, 2076 (2009)

15. R. Bernabei et al., Yadernaya Fizika 72, 2138 (2009)

16. S.K. Lee, M. Lisanti, B.R. Safdi, JCAP 11, 033 (2013)

17. K. Freese, M. Lisanti, C. Savage, Rev. Mod. Phys. 85, 1561 (2013). [arXiv:1209.3339]

18. C. McCabe, [arXiv:1312.1355]

19. P.J.T. Leonard, S. Tremaine, Astrophys. J. 353, 486 (1990)

20. C.S. Kochanek, Astrophys. J. 457, 228 (1996)

21. K.M. Cudworth, Astron. J. 99, 590 (1990)

22. J. Delhaye, Stars and Stellar Systems, vol. 5. (Univ. of Chicago Press, Chicago, 1965), p. 73

23. Starlink Project, http://starlink.jach.hawaii.edu/starlink

24. J. Lewin, P. Smith, Astropart. Phys. 6, 87 (1996)

25. W.T. Eadie et al., Statistical Methods in Experimental Physics. (American Elsevier Pub., Amsterdam, 1971)

26. G.J. Feldman, R.D. Cousins, Phys. Rev. D57, 3873 (1998)

27. M. Ambrosio et al., Phys. Rev. D 67, 042002 (2003) 